\title{
Antimalarial prenylated chalcones from the twigs of Dorstenia barteri var. subtriangularis
}

\author{
Bathelemy Ngameni $^{\mathrm{a}}$, Jean Watchueng ${ }^{\mathrm{a}}$, Fabrice Fekam Boyom ${ }^{\mathrm{b}^{*}}$, Felix Keumedjio ${ }^{\mathrm{a}}$, \\ Bonaventure T. Ngadjui ${ }^{\mathrm{a}}$, Jiri Gut ${ }^{\mathrm{c}}$, B.M. Abegaz ${ }^{\mathrm{d}}$, and Philip J. Rosenthal ${ }^{\mathrm{c}}$ \\ ${ }^{a}$ Department of Organic Chemistry, Faculty of Science, University of Yaoundé 1, P.O. Box 812, \\ Yaoundé, Cameroon \\ ${ }^{b}$ Department of Biochemistry, Faculty of Science, University of Yaoundé 1, P.O. Box 812, \\ Yaoundé, Cameroon \\ ${ }^{c}$ Division of Infectious Diseases, Department of Medicine, University of California San \\ Francisco, USA \\ ${ }^{d}$ Department of Chemistry, University of Botswana, Private bag 0022, Gaborone, Botswana \\ E-mail:ffefe@fulbrightweb.org
}

\begin{abstract}
The ever widening level of Plasmodium falciparum resistance to antimalarials has led to the search of alternative therapies. In this context, any resources that can help alleviate the burden of the deadly malaria; including the search for new plant-derived biologically active ingredients are worthy of investigation. Amongst these, naturally occurring and synthetic chalcones have demonstrated promising potencies. In the present study, two diprenylated chalcones Bartericin A 1 and B 2, and four known natural products, stipulin 3, 4-hydroxylonchocarpin 4, isobavachalcone 5 and kanzonol B 6 were isolated from the twigs of Dorstenia barteri var. subtriangularis (Moraceae) by means of chromatographic methods. The structures of the purified compounds were elucidated by spectroscopic methods, mainly 1D and 2D-NMR spectroscopy. These compounds (1-6) were evaluated in culture against the W2 strain of P. falciparum. The evaluated compounds were found to be active in vitro against $P$. falciparum, 1,3 and 4 demonstrating particular potencies with relatively low $\mathrm{IC}_{50}$ values $(2.15 \mu \mathrm{M}, 5.13 \mu \mathrm{M}$ and 3.36 $\mu \mathrm{M}$ respectively). The observed activities confirmed the chalcones as potential leads for the development of antimalarials.
\end{abstract}

Keywords: Dorstenia barteri, Moraceae, chalcones, Plasmodium falciparum, antiplasmodial activity 


\section{Introduction}

The morbidity and mortality associated with malaria have spurred efforts to find novel antiplasmodial agents with improved potency and selectivity. Leads for antimalarial agents continue to be isolated from natural sources and chemical syntheses. The structural diversity of compounds with micromolar and lower activities points to the considerable tolerance for different structural elements in the "antimalarial pharmacophore".

The antimalarial activity of chalcones, which are precursors of the better known family of flavonoids, came about through two approaches. In the first approach, routine screening resulted in the discovery of the antimalarial activity of licochalcone A $\left(\mathrm{IC}_{50}=1.8 \mu \mathrm{M}\right.$ against $P$. falciparum Dd2 strain), a substance found in the roots of the Chinese liquorice. ${ }^{2}$ At about the same time, a second approach consisted in the construction of a homology model of the malarial cysteine protease and its use as a template for a computational search of a database of commercially available small molecules that could fit the model of the active site. ${ }^{3}$ Modeling studies showed that both acyl hydrazides and chalcones could fit well into the putative active site.

Since then, a series of alkoxylated, hydroxylated, prenylated, oxygenated, quinolylated chalcones from natural sources and syntheses have been evaluated for antiplasmodial activity with encouraging results. ${ }^{4-10}$

From our continuing search for a new alternative cure for malaria, we report in this paper the antiplasmodial activities of six chalcones 1-6 isolated from the extract of the twigs of Dorstenia barteri var. subtriangularis (Moraceae).

\section{Results and Discussion}

\section{Isolation and characterization of compounds}

The polar fraction of the organic extract of the twigs was filtered through a Sephadex LH-20 column, followed by repeated silica gel CC and preparative TLC to give six compounds, 1 - 6 (figure 1). Four known prenylated chalcones 3, 4, 5 and $\mathbf{6}$ were identified as stipulin, 4hydroxylonchocarpin, isobavachalcone and kanzonol B respectively, by direct NMR spectral data analysis and comparison with literature data. ${ }^{11-13}$ Structure elucidation of Bartericin A $\mathbf{1}$ and B 2 was recently reported from our previous study. ${ }^{14}$ 
<smiles>[R]c1cc(/C=C/C(=O)c2cc([R])c(O)c([R])c2O)ccc1O</smiles><smiles>[R]C/C=C(/C)C(=O)CC(O)C=[R]</smiles>
$3 \mathrm{R}^{1}=\mathrm{H}, \mathrm{R}^{2}=$ 入 $\mathrm{R}^{3}=\widehat{ح}$<smiles>CC(C)=CCc1cc(C=CC(=O)c2cc3c(cc2O)O[C-](C(C)(C)O)[CH-]3)ccc1O</smiles><smiles>CC(C)=CCc1c(O)ccc(C(=O)/C=C/c2ccc(O)cc2)c1O</smiles>

Figure 1. Structures of the compounds (1-6) isolated from Dorstenia barteri var. subtriangularis.

\section{Antiplasmodial activities of isolated compounds 1-6}

Test compounds 1-6 showed toxicity to erythrocytes at concentrations above $20 \mathrm{mM}$, about three orders of magnitude above concentrations with antimalarial activity. Compounds were tested for their antiplasmodial activity against the W2 strain of $P$. falciparum, which is resistant to chloroquine and other antimalarials.

All six compounds 1-6 were found to be active against strain W2 of $P$. falciparum in culture. The most active compounds were $\mathbf{1}, \mathbf{3}$, and 4 , with respective $\mathrm{IC}_{50}$ values of $2.15 \mu \mathrm{M}, 5.13 \mu \mathrm{M}$ and $3.36 \mu \mathrm{M}$ (figure 2 and table 1 ). 

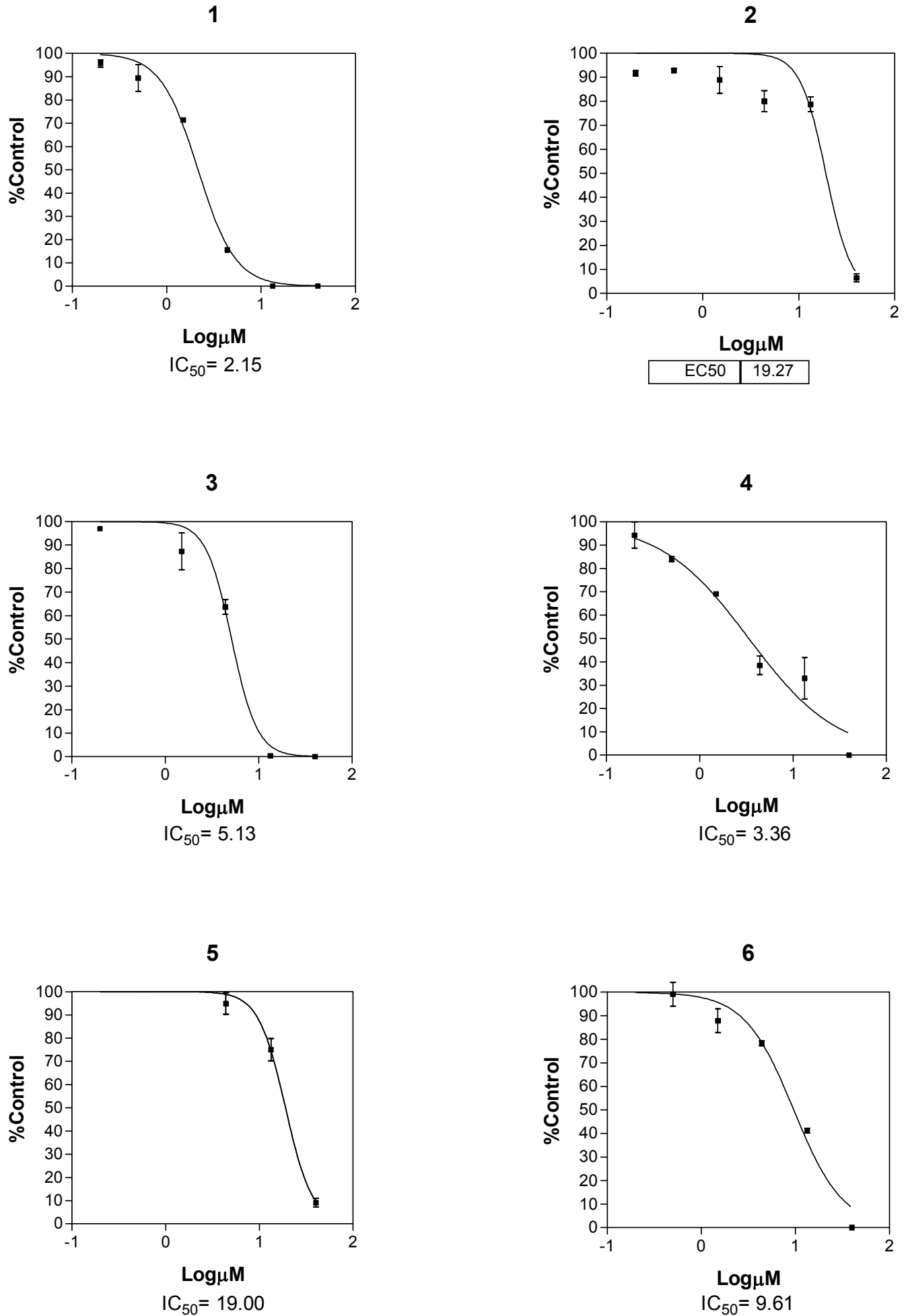

Figure 2. Sigmoidal dose-response curves of compounds 1-6. 


\section{Caption to Figure 2}

Compounds were diluted and incubated with cultured W2-strain $P$. falciparum parasites for $48 \mathrm{~h}$, parasites were fixed and stained, and parasitemias of treated and control cultures were determined. Results are means, compared to untreated controls, from three experiments. Error bars represent standard deviations of results.

Table 1. $\mathrm{IC}_{50}$ values of evaluated compounds 1-6 against strain $\mathrm{W} 2$ of $P$. falciparum in culture

\begin{tabular}{rlrrrrrr}
\hline Compound & \multicolumn{1}{c}{$\mathbf{1}$} & $\mathbf{2}$ & $\mathbf{3}$ & $\mathbf{4}$ & $\mathbf{5}$ & $\mathbf{6}$ & $\mathbf{C Q}$ \\
\hline $\mathrm{IC}_{50}{ }^{a} \pm \mathrm{SD}(\mu \mathrm{M})$ & $2.15 \pm$ & $19.27 \pm$ & $5.13 \pm$ & $3.36 \pm$ & $19.00 \pm$ & $9.61 \pm$ & $0.13 \pm$ \\
& 0.02 & 0.06 & 0.04 & 0.07 & 0.02 & 0.04 & 0.02 \\
Erythrocytes & & & & $>20 \mathrm{mM}$ & & & \\
susceptibility & & & & & & & \\
\hline
\end{tabular}

${ }^{a}$ Concentration that killed $50 \%$ of parasites relative to negative control. $\mathrm{SD}=\mathrm{Standard}$ Deviation; the compounds were tested in triplicate. $\mathrm{CQ}=$ chloroquine

From the study of the structure-activity relationships, it appears that the presence of a hydroxylated prenyl group on carbon 5' (Ring B) enhances the antiplasmodial activity of compound 1 compared to compound $\mathbf{3}$ that bears a simple prenyl group at the same position. Furthermore, the cyclic pyran prenyl group at carbon 3' confers significant activity to compound 4 compared to compound 5 that bears a prenyl group at the same position. On the other hand, the hydroxylated cyclic furan prenyl at carbon 5' in compound $\mathbf{2}$ considerably impairs its antiplasmodial activity relative to compounds $\mathbf{1}$ and $\mathbf{3}$ that are more potent.

These results corroborate the potency of chalcones that have already been shown to inhibit some metabolic pathways in Plasmodia, and especially the cysteine proteases' activity. ${ }^{1,3,7}$

From a recent investigation, Narender et al. (2005) ${ }^{4}$ isolated Medicagenin, a diprenylated chalcone (figure 3) from the roots of Crotalaria medicagenia (Leguminosae) that exhibited good potency against strain NF-54 of $P$. falciparum $\left(\mathrm{IC}_{50} \approx 5 \mu \mathrm{M}\right)$. The activity of our compound 3 is comparable to that of the above derivative; more interestingly, our compounds $\mathbf{1}$ and $\mathbf{4}$ were found to be more active in vitro against chloroquine resistant W2 strain of $P$. falciparum, with respective $\mathrm{IC}_{50}$ values of $2.15 \mu \mathrm{M}$ and $3.36 \mu \mathrm{M}$.

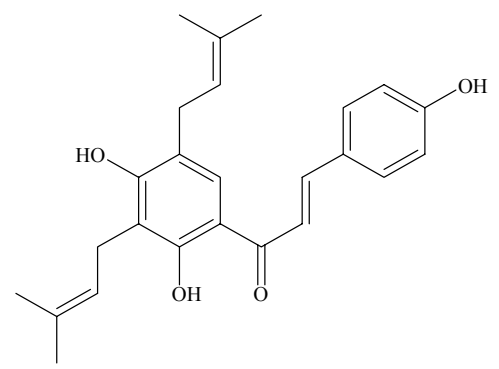

Figure 3. Structure of Medicagenin isolated from the roots of Crotalaria medicagenia (Narender et $a l ., 2005)$. 


\section{Experimental Section}

\section{Plant material}

The twigs of D. barteri var. subtriangularis were collected at Tombel in the South-western province of Cameroon in November 2001. The plant was identified by Mr. Victor NANA from the National Herbarium in Yaoundé, where a voucher specimen ( $\mathrm{N}^{\mathrm{O}}$ 19534/SRF Cam.) was deposited.

\section{Extraction and compound isolation}

Air-dried and powdered twigs (450 g) were successively macerated with a mixture of $\mathrm{CH}_{2} \mathrm{Cl}_{2}$ $\mathrm{MeOH}(1: 1)$ and $\mathrm{MeOH}$ for 24 and 2 hours, respectively, at room temperature. These two crude extracts were combined (70 g) based on their thin layer chromatography (TLC) patterns. $65 \mathrm{~g}$ of this organic extract was subjected to column chromatography (CC) on silica gel 60 (200 g) and eluted with petroleum ether (60/80) followed by pet. Ether-EtOAc $(3: 1,1: 1,1: 3)$ mixtures and then EtOAc to give fractions A to $\mathrm{E}$ of $500 \mathrm{ml}$ each. The fraction A $(5 \mathrm{~g})$ eluted with pet. Ether contained mainly mixtures of oils and was not investigated further. Fraction B (30.2 g) was passed through Sephadex LH-20 column and eluted with $\mathrm{CHCl}_{3}-\mathrm{MeOH}$ (2:1). The post chlorophyll fraction (10.5 g) was subjected to silica gel 60 (150 g) CC separations and eluted with hexane followed by hexane-EtOAc gradient. 25 fractions of $250 \mathrm{ml}$ each were collected and combined on the basis of TLC patterns. Fractions 1-5 eluted with hexane-EtOAc (95:5) yielded 4-hydroxylonchocarpin 4 (25 mg). Fractions 6-12 obtained with hexane-EtOAc $(9: 1,4: 1)$ gave isobavachalcone $5(15 \mathrm{mg})$. Fractions 13-25 obtained with hexane-EtOAc (7:3) were combined to give $3 \mathrm{~g}$ of a mixture of three compounds from the TLC patterns; part (1g) of this mixture was purified by preparative TLC using $\mathrm{CHCl}_{3}-\mathrm{MeOH}$ (97:3) to give stipulin 3 (40 mg); bartericin A 1 (35 mg) and B 2 (40 mg). Combined fractions C - E (14.5 g) were also passed through Sephadex $\mathrm{LH}-20$ and eluted with $\mathrm{CHCl}_{3}-\mathrm{MeOH}(2: 1)$; the post chlorophyll fractions $(2 \mathrm{~g})$ was subjected to $\mathrm{CC}$ (silica gel, $50 \mathrm{~g}$ ) using $\mathrm{CH}_{2} \mathrm{Cl}_{2}-\mathrm{MeOH}$ (95:5) to afford kanzonol B 6 (20 mg) and bartericin A 1 (5 mg).

The structures of the purified compounds were elucidated by spectroscopic methods, mainly 1D and 2D-NMR spectroscopy. ${ }^{14}$

\section{Evaluation of erythrocyte susceptibility to compounds 1-6 in vitro}

A preliminary toxicological assessment was carried out to determine the highest drug concentrations that can be incubated with erythrocytes without any significant damage. This was done according to the 3-[4,5-dimethylthiazol-2-yl]-2,5-diphenyltetrazolium bromide/phenazine methosulfate (MTT/PMS) colorimetric assay described by Cedillo-Rivera et al. (1992), ${ }^{15}$ with some modifications. The drugs were serially diluted in 96 well culture plates, and each concentration incubated in triplicate with erythrocytes (2\% hematocrit) in a final $100 \mathrm{ml}$ culture volume (at $37^{\circ} \mathrm{C}$, in a $3 \% \mathrm{O}_{2}, 5 \% \mathrm{CO}_{2}$ and $91 \% \mathrm{~N}_{2}$ atmosphere, in the presence of RPMI 1640, $25 \mathrm{mM}$ HEPES, pH7.4 for $48 \mathrm{~h}$ ). At the end of the incubation period, the cultures were 
transferred into polypropylene microcentrifuge tubes and centrifuged at $1500 \mathrm{rpm}$ for $5 \mathrm{~min}$, and the supernatant was discarded. $1.5 \mathrm{ml}$ MTT solution with $250 \mathrm{~m} \mathrm{~g}$ PMS were added to the pellets. Controls contained no erythrocytes. The tubes were thereafter incubated for $45 \mathrm{~min}$ at $37{ }^{\circ} \mathrm{C}$, then centrifuged, and the supernatant was discarded. The pellets were re-suspended in $0.75 \mathrm{ml}$ of $\mathrm{HCl} 0.04 \mathrm{M}$ in isopropanol to extract and dissolve the dye (formazan) from the cells. After 5 min, the tubes were vigorously mixed and centrifuged, and the absorbance of the supernatant was determined at $570 \mathrm{~nm}$.

\section{Evaluation of antiplasmodial activity}

$P$. falciparum strain W2, which is resistant to chloroquine and other antimalarials, ${ }^{16}$ was cultured in sealed flasks at $37^{\circ} \mathrm{C}$, in a $3 \% \mathrm{O}_{2}, 5 \% \mathrm{CO}_{2}$ and $91 \% \mathrm{~N}_{2}$ atmosphere in RPMI 1640, $25 \mathrm{mM}$ HEPES, pH 7.4, supplemented with heat inactivated 10\% human serum and human erythrocytes to achieve a $2 \%$ hematocrit. Parasites were synchronized in the ring stage by serial treatment with $5 \%$ sorbitol (Sigma) ${ }^{17}$ and studied at $1 \%$ parasitemia.

Compounds were prepared as $10 \mathrm{mM}$ stock solutions in DMSO, diluted as needed for individual experiments, and tested in triplicate. The stock solutions were diluted in supplemented RPMI 1640 medium so as to have at most $0.2 \% \mathrm{DMSO}$ in the final reaction medium. An equal volume of $1 \%$ parasitemia, $4 \%$ hematocrit culture was thereafter added and gently mixed thoroughly. Negative controls contained equal concentrations of DMSO. Positive controls contained $1 \mu \mathrm{M}$ chloroquine phosphate (Sigma). Cultures were incubated at $37^{\circ} \mathrm{C}$ for $48 \mathrm{hrs}$ (1 parasite erythrocytic life cycle). Parasites at ring stage were thereafter fixed by replacing the serum medium by an equal volume of $1 \%$ formaldehyde in PBS. Aliquots $(50 \mu 1)$ of each culture were then added to $5 \mathrm{ml}$ round-bottom polystyrene tubes containing $0.5 \mathrm{ml} 0.1 \%$ Triton X-100 and 1 nM YOYO nuclear dye (Molecular Probes) in PBS. Parasitemias of treated and control cultures were compared using a Becton-Dickinson FACSort flow cytometer to count nucleated (parasitized) erythrocytes. Data acquisition was performed using CellQuest software. These data were normalized to percent control activity and 50\% inhibitory concentrations ( $\mathrm{IC}_{50}$ values) calculated using Prism 3.0 software (GraphPad) with data fitted by non linear regression to the variable slope sigmoidal dose-response formula:

$\mathrm{y}=100 /\left[1+10^{(\log \mathrm{IC} 50-x) H}\right]$, where $H$ is the hill coefficient or slope factor. ${ }^{16}$

\section{Conclusions}

The synthetic design of chalcones as antimalarial agents was based primarily on their potential to inhibit malarial cysteine protease, but it is likely that other factors (interference with glutathione mediated breakdown of Falcipain, for example) besides falcipain inhibition contribute to their antimalarial activity. ${ }^{1}$ The results obtained from this investigation highlight the fact that further investigations are needed to elucidate the real bioactivity pathways of these compounds, together with more emphasis on the search for naturally occurring potent derivatives. Moreover, the 
results achieved in this investigation highlight the chalcones as potential leads for the development of antimalarials.

\section{Acknowledgements}

This investigation was supported by IPICS grants (under the auspices of NABSA) at the University of Botswana and the National Institute of Health, USA (AI35707). We gratefully acknowledge the practical help of Mr. Nana of the National Herbarium for his assistance for the collection and identification of plant material

\section{References and Footnotes}

1. Go, M. L. Med. Res. Rev. 2003, 23, 456.

2. Chen, M.; Theander, T. G; Christensen, S. B; Hviid, L.; Zhai, L.; Kharazmi, A. Antimicrob. Agents Chemother. 1994, 38, 1470.

3. Li, R.; Chen, X.; Gong, B.; Selzer, P. M.; Li, Z., Davidson, E.; Kurzban, G.; Miller R. E.; Nuzum, E. O.; McKerrow, J. H.; Fletterick, R. J.; Gillmore, S. A.; Craik, C. S.; Kuntz, I. D.; Cohen, F. E.; Kenyon, G. L. Bioorg. Med. Chem. 1996, 9, 1421.

4. Narender, T.; Shweta, K.; Tanvir, M.; Srinivasa, R. K.; Srivastava, S. K.; Puri, S. K. Bioorg. Med. Chem. Lett. 2005, 15, 2453.

5. Go, M. L.; Liu, M.; Wilairat, P.; Rosenthal, P. J.; Saliba, K. J.; Kirk, K. Antimicrob. Agents Chemother. 2004, 48, 3241.

6. Liu, M.; Wilairat, P.; Go, M. L. J. Med. Chem. 2002, 45, 1734.

7. Dominguez, J. N.; Charris, J. E.; Lobo, G.; Gamboa, D. N.; Moreno, M. M.; Riggione, F.; Sanchez, E.; Olson, J.; Rosenthal, P. J. Eur. J. Med. Chem. 2001, 36, 555.

8. Ram, V. J.; Saxena, A. S.; Srivastava, S.; Chandra. Bioorg. Med. Chem. Lett. 2000, 10, 2159.

9. Chen, M.; Brogger, C. S.; Zhai, L.; Rasmussen, M. H.; Theander, T. G.; Frokjaer, S.; Steffansen, B.; Davidsen, J.; Kharazmi, A. J. Infect. Dis. 1997, 176, 1327.

10. Li, R.; Kenyon, G. L.; Cohen, F. E.; Chen, X.; Gong, B.; Dominguez, J. N.; Davidson, E.; Kurzban, G.; Miller, R. E.; Nuzum, E. O. J. Med. Chem. 1995, 38, 5031.

11. Abegaz, B. M.; Ngadjui, B. T.; Dongo, E.; Tamboue, H. Phytochemistry 1998, 49, 1147.

12. Monache, G. D.; Mello, J. F. D.; Monache, F.; Bettolo, G. B. M.; Lima, O. G. D.; Coelho, J. S. D. B. Grazz. Chim. Ital. 1974, 104, 861.

13. Fukai, T.; Nishizawa, J.; Nomura, T. Phytochemistry 1994, 35, 515.

14. Ngameni, B., Ngadjui, B. T.; Folefoc, G. N.; Watchueng, J.; Abegaz, B. M. Phytochemistry 2004, 65, 427.

15. Cedillo-Rivera, R., Ramfrez, A., Munoz, O. Arch. Med. Res. 1992, 23, 59.

16. Singh, A.; Rosenthal, P. J. Antimicrob. Agents Chemother. 2001, 45, 949.

17. Lambros, C.; Vanderberg, J. P. J. Parasitol. 1979, 65, 418. 